\title{
Coming in from the cold. From psychiatric to general hospital. The Irish experience
}

\author{
DERMOT WALSH
}

\begin{abstract}
The metamorphosis from an extensive mental hospital system of care, rooted in a culture and tradition of self-sufficiency and isolation, to the concept and practise of delivery of psychiatric care in general hospitals is described. The obstacles, psychological and practical, to be overcome in this change process are outlined. The place of the general hospital psychiatric unit in psychiatric and general medical care is outlined. Relevant matters of design and management are briefly explored.
\end{abstract}

\section{CARE IN IRELAND 1750 - 1900}

In common with other Western European countries the public visibility of mental illness in Ireland became apparent towards the middle of the $18^{\text {th }}$ century. The reasons for this are unclear but it may have been that hitherto those mentally ill, or likely to become so, experienced poorer survival to adulthood than those not so vulnerable. Because decreased premature mortality and improved life expectation for entire populations, mainly because of better nutrition, transformed European demography, mental illness obtruded as had not previously been the case. Whether or not this postulated effect was allied to or independent of the possibility of an increased incidence of major psychotic illness in the late $18^{\text {th }}$ century by other mechanisms must remain an open question. Another influence invoked to account for the "apparent" increase, urbanisation, hardly applied in Ireland which remained very largely an agricultural economy. However that may have been, one of the earliest responses to this increased evidence of "lunacy" in Ireland, which had begun to impact on public order and resulted in many mentally ill persons being incarcerated in prisons as well as being "at large", was the bequeathing of monies by Jonathan Swift, the celebrated churchman and author of Gulliver's Travels, to provide a specialist hospital for the mentally ill poor in Dublin:

He gave the little wealth he had

To build a house for fools and mad

Address for correspondence: Dr. D. Walsh, Health Research Board, Knockmaun House, Lower Mount Street, Dublin, 2 (Ireland).

Fax: +353-6611856

E-mail: dwalsh@hrb.ie

Declaration of Interest: none.

\section{And showed by one satiric touch \\ No nation wanted it so much}

The hospital, known initially as Swift's Hospital, later to become St. Patrick's, opened in 1757. At the outset it catered for the "lunatic poor" as Swift had specified but as time went on, increasingly, for financial survival, accepted private patients. When this practise was challenged the founding charter was altered to allow the hospital to accept paying patients and today it is an entirely private hospital (Malcolmson, 1989).

Also in the $18^{\text {th }}$ century further provision was made for the mentally ill in both private and public accommodation in a haphazard fashion in the cities of Dublin and Cork. Nevertheless the problem of the unaccomodated mentally ill continued to increase to the extent that legislation of 1821 led to the setting up of a national system of district lunatic asylums based on catchments of combinations of cities and counties, so that by 1840 there were 11 such asylums providing for 2,228 patients representing 28.7 beds $/ 100,000$ population - a population of the island of Ireland ( 32 counties), which at the census of 1831 , stood at $7,767,401$.

Despite this initiative, large numbers of mentally ill deemed in need of asylum care remained in workhouses (institutions maintained for the care of the indigent poor and others with a variety of social problems), prisons or " at large". Accordingly further provision of new asylums, and the enlargement of those existing, was set in train. In the planning of the quantative requirement for additional asylum places, the police were invoked to carry out a prevalence count of those mentally ill "at large" in each district, and on the results of this and the known numbers in other institutions, building proceeded. Finally around 1900 the job was done, as policy had dictated it should be, and virtually all ascertained mentally 
ill were in 24 specialist stand -alone district lunatic asylums or, in much smaller numbers, in private hospitals, to the tune of 16,500 persons, representing over one third of one per cent of a population reduced by famine and emigration to four and one half million by 1901 .

\section{CARE FROM 1900 - 2005}

With the formation of the Irish Free State in 1922, subsequently to become Ireland or the Republic of Ireland, jurisdiction was reduced to 26 counties with the remaining six counties, known as Northern Ireland, remaining within the United Kingdom.

In the Republic nothing much changed during the first half of the twentieth century other than the number of persons in the mental hospitals increased to 21,000 by 1958 or 750 per 100,000 of the population which now numbered less than $3,000,000$. This represented a far higher hospitalisation rate than prevailed elsewhere and included in this number were over 2,600 intellectually disabled persons, accommodated in psychiatric hospitals because of inadequate specialised provision for them elsewhere. Those suffering from mental illness requiring inpatient treatment had up to now no alternative, in the absence of general hospital provision, other than to enter a grim $19^{\text {th }}$ century asylum

This and other shortcomings were acknowledged in the Report of the Commission of Enquiry on Mental Illness (Department of Health, 1966). This report recommended, inter alia, that "the needs of short-term patients can best be met by psychiatric units in, or associated with, general hospitals". In fact, the first such unit opened in 1967 and by 1984 there were 10 units providing 424 of the total psychiatric bed numbers which by now had fallen to 12,800. In that year another Government report on the future of psychiatric services issued and indicated that a further 386 beds would shortly be provided in psychiatric units in nine further general hospitals (Department of Health and Children, 1984). It additionally recommended that all such units should provide psychiatric liaison services to the general hospitals in which they were based.

By the end of 2005, when inpatient numbers had fallen to approximately 3,000, of which all but 500 were in the public sector, there were 22 general hospital psychiatric units accounting for over $60 \%$ of public hospital admissions and providing just short of 900 beds (that equates to 2.3 beds for 10,000 general population). In addition there remained a further 400 acute admission beds in psychiatric hospitals in those 10 catchment services without a general hospital unit. The remaining,
1,200 public beds in the remnants of the 13 or so psychiatric hospitals which had not entirely closed, were occupied by long-stay patients awaiting community relocation. The two private psychiatric hospitals catering for acute illness remained free-standing. While there is a small, but growing number of private general hospitals, none of these have psychiatric units. All existing general hospital units serve the catchments originally allocated to their services with the setting up of the district asylum system and, on average, cater for catchments of 100,000 population, although the range is from 50,000 to 250,000

\section{SMALL HOSPITALS AND LOCAL}

Ireland has historically been a country of small hospitals with strong local identities and allegiances but it has long been recognised that, efficiency apart, modern health care delivery requires larger, multi-specialty hospitals serving larger populations. In fact this reality was the basis of a Government report on the Outline of the General Hospital System, which, in 1968, recommended the rationalisation of the system leading to the closure of smaller hospitals and the concentration of expertise, skills and specialties in larger centres (Department of Health, 1968). Attempts to implement these recommendations were opposed by local community groups fearful of losing their hospital with the consequence that, although some progress was made, major problems remained. A further, more recent, report of 2004 reinforced the necessity of changes along the lines recommended in 1968 and the putting in place of acute general hospital catchments areas of 350,000 population centred on 13 or so enhanced, mainly university- related, general hospitals ( Department of Health and Children 2003).

\section{LARGER HOSPITALS AND CATCHMENT AREAS}

In concert a major Government report on psychiatric services earlier this year advocated that all inpatient psychiatric care should be based in these 13 general hospitals, the majority of which already have psychiatric units. These units would deliver acute inpatient and community care to these same 350,000 catchments thus providing multi-specialty mental health services to these populations, something not possible in the smaller existing catchments of which there are currently 31 . In recommending a major strengthening of community services to include and expand crisis intervention, home care, early intervention and assertive outreach services, the report 
envisaged that 650 general hospital -based beds (that equates to 1.6 beds for 10,000 general population), rather than the existing 1,300 acute beds, would suffice (Department of Health and Children, 2006). Given the current over-supply of units and beds based on 100,000 population catchments, the shift to larger catchments will result in a reduction from the 22 existing units to a nominal 13. A new role for these redundant units will be found, such as their functional conversion to community mental health centre status, perhaps including crisis overnight accommodation. While there is optimism that the necessary capital and revenue funding to establish the proposed expanded community developments will be forthcoming, support will also be necessary to upgrade some of the 13 units chosen for the future acute inpatient service, as some of these date back over 30 years and need adaptation for satisfactory modern acute inpatient care.

Moving from a traditional isolated self-sufficient, stand- alone psychiatric setting, with a patina of tradition and cultural values centuries old, requires courage and change management and this has been very evident in the Irish experience. The moves have required lengthy and wearing negotiations with staff representative bodies particularly with staff fearful of merging with the wider world of general hospital activity. Relocation has often been, literally, a costly matter in a country where. 60 years or so ago, the local mental hospital might have been the largest employer in a country town.

\section{ADMINISTRATIVE AND MANAGEMENT ISSUES}

The relationship of the general hospital unit to the administrative framework of the general hospital, while at the same time maintaining and relating to its community service component, has raised issues of autonomy, traditionally sacrosanct, particularly in staff reporting relationships, which have not been without their own impact. With skill in negotiation a workable balance can be struck in the matter of "serving two masters" (the catchment psychiatric service and the general hospital). Here the provision of a comprehensive and readily available liaison service to the general hospital of which one is a part and to its accident and emergency department, will go a long way towards earning respect and creating capacity for working together. And liaison is a two-way process, readily accessible consultation to the psychiatric unit from medical and surgical teams, when required, is a further benefit of being in a general hospital setting

Successful design is crucial to successful function. From the outset the parameters of care have to be identified and agreed. Ideally the unit should be capable of caring for and treating all acute psychiatric illness efficiently and safely - and safety here concerns staff as well as patients while respecting personal integrity and dignity. Implicit in this is the precept that users and carers are integral members of the care and treatment process. Risk assessment will guide the limits of safe care and treatability.

In this context one of the issues to be faced is the adequate provision for segregation of the sexes with some stepping back from the concept of integration of earlier years which was an understandable countervailing consideration to the absolute isolation and unreal separation of the sexes in the old mental hospital. The issue of disturbed behaviour in the general hospital setting requires consideration. While in general admission, and the procedures associated with it, will be managed as admission to the hospital generally, there will, infrequently, be very disturbed patients requiring admission expediously without proceeding through the main hospital concourse and emergency room and for these unusual circumstances direct admission to the unit will be necessary; for this purpose the unit should have its own separate entrance. In some instances a unit may be free-standing on the general hospital campus and this will be less of a problem. In others, and this is generally seen as the better arrangement, the unit will be within the fabric of the general hospital as a whole and separate and discreet access may pose more of a difficulty. In some services there may be an edict that all new referrals should be triaged through and be physically examined in, the accident and emergency department to ensure that an apparently psychiatric presentation does not have an underlying somatic cause more appropriately dealt with on the medical or surgical wards.

\section{DESIGN ISSUES}

Within the unit itself debate often centres on whether a self contained "high observation" sub-unit or section is necessary and if so what nature it should take. Obviously in a unit of 35-50 beds or so overall, accommodation of this nature for four patients or so at any one time will be sufficient. Questions that will be asked include should this component be locked from the main unit and should all activity, including taking meals, be catered for within its confines. Should it have one or two "safe" rooms, if necessary, for seclusion purposes, and when this is the case are there documented and agreed protocols concerning the management of the sub-unit and the "safe" rooms, including who decides when a patient should be sent thereto, and governing their subsequent monitoring and 
supervision. Some may feel that such sub-unit high observation provision is essential, others that it creates ghettoisation and may be utilised for "punishment" purposes and is therefore reprehensible. Many will no doubt argue that the sub-unit arrangement entails increased staffing requirements. Will such a provision do away with the locking of the main unit where this is ordinarily the practise? And there may be some instances where, in the light of serious and potentially damaging behaviour, the principle of "treat all" may have to be retracted so that a very few patients are deemed unsuitable for safe care in the general hospital without recourse to a regional secure care and treatment unit, in most cases for a very short stay while major disturbance abates. In some jurisdictions legislation may empower courts to send patients to units for assessment and/or treatment and here clinicians may experience ethical and practical conflict between custodial and llibertanian considerations.

\section{CATERING FOR SUB-SPECIALITIES}

The question as to how general hospital units should cater for psychiatric sub-specialities has also to be broached. Should all units have separate sub-units of, say, four beds for the assessment and acute treatment of the elderly, particularly where, in the catchment there is, as there should be, a specialist team for the psychiatric illness of later life. As this is a major subspecialty such provision will be required in all units serving catchments of 350,000 or so. In contrast a much more limited provision may be required for the more "rarified" subspecialties such as that of adult eating disorders, on a regional rather than on a catchment basis. And the specialised catchment team dealing with the psychiatric illnesses of the intellectually disabled may also require a bed from time to time to deal with the acute and time-limited illnesses of their patients from the catchment who are suitable for treatment in such units.

\section{STAFFING}

Who staffs the general hospital psychiatric unit? Many disciplines obviously. But are these the same personnel who deliver the community services or are they exclusive to the unit. The principle of continuity of care enjoins that staff in the community where, after all, the majority of care is delivered, follow their patients into inpatient care and there are many cogent reasons why this is the preferred model in care management and the one that users and carers prefer. The concept of a "hospitalist", as in some general medical and surgical settings, cuts across too many principles to be adopted in inpatient psychiatric settings. Notwithstanding, there will be calls for a "bed manager" nominated by the clinical teams to ensure that all admissions are necessary and that discharge is not unduly delayed as had been the experience in Ireland where almost half of acute beds were occupied by patients whose illness was no longer acute and for whom appropriate community alternatives were the better option (Keogh et al 1999).

\section{CONCLUSIONS}

In conclusion the recognition that persons with psychiatric illness are entitled to the same rights and privileges in care and treatment as those with other illnesses, and in the same setting, is a fundamental tenet of service delivery philosophy, nowhere better symbolised than by the shift, often painful but always worthwhile, from psychiatric hospital to general hospital psychiatric unit. Among other advantages is the increased awareness and acknowledgment by medical and surgical departments of the importance of psychiatric morbidity in its own right and in its contribution to physical illness. The responsibility of the liaison psychiatric function in promoting this awareness is self-evident. Furthermore, in a wider sense, psychiatry becomes part of the general hospital, not least in under- and post-graduate education and research.

\section{REFERENCES}

Department of Health (1966). Report of the Commission of Enquiry on Mental Illness. Stationery Office: Dublin.

Department of Health (1968). Outline of the Future Hospital System. Report of the Consultative Council on the General Hospital Services. Stationery Office: Dublin.

Department of Health (1984). The Psychiatric Services. Planning for the Future. Stationery Office: Dublin.

Department of Health and Children (2003). Task Force on Medical Manpower. Stationery Office: Dublin.

Department of Health and Children (2006). A Vision for Change. Stationery Office: Dublin.

Keogh F. Roche A. \& Walsh D. (1999). We Have No Beds. Health Research Board: Dublin.

Malcolmson E. (1989). Swift's Hospital. Gill and Macmillan: Dublin. 